\title{
The Blockchain in Microgrids for Transacting Energy and Attributing Losses
}

\author{
E. Riva Sanseverino ${ }^{1}$, M. L. Di Silvestre ${ }^{1}$, P. Gallo ${ }^{1,2}$, G. Zizzo ${ }^{1}$, and M. Ippolito ${ }^{1}$ \\ ${ }^{1}$ DEIM - University of Palermo, Italy \\ ${ }^{2}$ CNIT - Italy
}

\begin{abstract}
In recent years novel models for energy distribution appeared and islanded microgrids quest for new ways to exchange energy between consumers and producers without the need of central authorities. The blockchain mechanism has emerged as a distributed solution for recording energy transactions in power systems. The blockchain has been used to permit users bartering and selling energy and to keep track of such exchanges without exposing them to tampering. In this work, we consider a novel application of the blockchain in islanded microgrids that includes also annotating energy losses caused by energy transactions, in order to have a more realistic matching between the physical status of the energy grid and the consequent costs attributed to users. To validate our novel use of the blockchain, we carried out simulated experiments for an exemplary islanded microgrid, in which 3 main generators supply 6 load nodes. This validates the compatibility of this new cost attribution model with the supporting physical infrastructure. Preliminary results demonstrate that the superposition of energy transactions in a microgrid changes the distribution of losses in all paths, eventually due to the large reactive flows created by PV systems.

Index Terms-Transactive energy; Energy blockchain; Microgrids.
\end{abstract}

\section{INTRODUCTION}

The advent of transactive energy as one of the most relevant technologies in the Gartner Hypecycle [1] and the birth of several startups around new cryptocurrency technologies demonstrate the rising interest around certified transactions on the Internet, without the need of trusted third parties[2]. Recent microgrid projects in the Brooklyn district of New York City use the blockchain technology for managing energy transactions and provide insights about a new energy system. This new energy system is based on distributed generation, energy trading between neighbors, and a novel role for distribution utilities. The energy market requires drastic reduction of the time spent for managing economic transactions due to energy trade. Furthermore, in islanded scenarios energy exchanges and their payments should not rely on central authorities. Using cryptocurrencies for monetary transactions is only one of the possibilities offered by blockchain in the energy field. Although this movement is currently largely being triggered by startups, utilities are catching up in these applications of energy blockchain and are starting joint ventures and cooperation. The basic value that new companies show to potential customers and investors is quite similar to that of initiatives in the bank sector. Any need for an intermediary between two parties is removed: switching to a decentralized energy system, detaching the related financial transactions from a centralized control unit, can be considered as another step towards a full decentralization. The case for energy is slightly different from other goods exchange. There are strong physical limitations and constraints that would reduce the realistic possibility of energy exchanges. In this aim, many companies have recently set up energy exchange platforms to aggregate buyers and sellers. As an example the dutch company Vandebron [3] offers the possibility to buy energy directly from producers using a central entity that manages the network, prepares billis and checks the balance between production and consumption. In microgrids, decentralized regulation offers the opportunity to manage effectively the blockchain technology for energy transactions. This paper presents the principles of the blockchain, proposes a novel application for energy transaction in residential microgrids and provides an exemplary set of energy transactions between nodes verifying their technical feasibility. Transactions occur in a given time frame and by tracking the power flows the physical evolution of the microgrid is monitored. Our preliminary experiments show that end-to-end opportunistic transactions become critical when loads and generation are separated by an infrastructure (i.e. a MV microgrid). In this case, large reactive flows are needed for voltage support. Moreover by employing the proposed algorithm, the active losses (and their monetization) can be exactly attributed to transactions (and therefore to users).

\section{The ENERGy BlockCHAIN}

In this section, the blockchain technology is briefly outlined, describing its basic principles and how it is tailored to address energy transactions for microgrids.

Blockchains are distributed databases that maintain ordered lists of records and permit transactions between peers without intermediary institutions (i.e. banks). Many cryptocurrencies, including Bitcoin, are based on the blockchain technology, which can also be applied in non-monetary transactions. For example, smart contracts are enabled by the blockchain and are automatically executed once specific conditions are fulfilled. Blockchains operate as distributed ledgers that contain a continuously growing list of data records called blocks [4]. Blocks are time-stamped, shared, unalterable and connected to preceding blocks as in a chain. Blocks can contain data, 


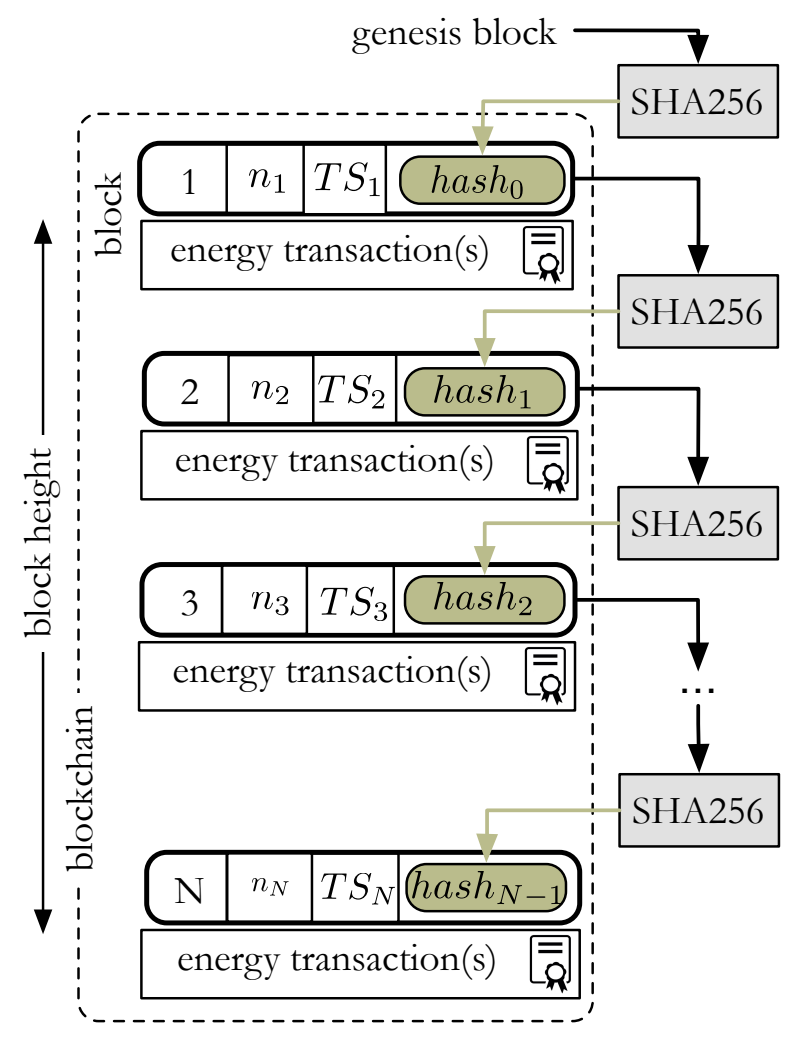

Fig. 1: Simplified conceptual scheme of an energy blockchain.

batches of individual transactions and eventually programs. In our scenario the blockchain allows users keeping track of all energy transactions that occur in the network. All users maintain a copy of the blockchain and can verify their blocks. Once transactions are included in blocks, they cannot be modified anymore because any tampering is revealed by the verification mechanism. The blockchain technology is enabled by the following elements:

- a verification mechanism;

- a data network to share the ledger.

Users can check that all blocks have not been subjected to tampering, quickly and efficiently, by checking only the last block. A data network is needed to permit prosumers sharing the distributed ledger. Fig. 1 shows the blockchain as a ledger of blocks, where each block contains one or more transactions. In case of energy purchase or selling, blocks can be organized in tables containing details including source (generator), destination (load), transferred energy [kWh], timestamp, duration, power profile $[\mathrm{kW}]$. We propose to add in the blockchain also presumed and/or measured losses due to non-linear effects and reactive power flows.

Each block in the blockchain contains a header and a data field. The header contains a string that uniquely identifies the block and is obtained from the previous block using the Secure Hash Algorithm SHA256 [5]. This is used to check for validity. The SHA algorithms receives in input a variable length message and produces a message digest, a footprint

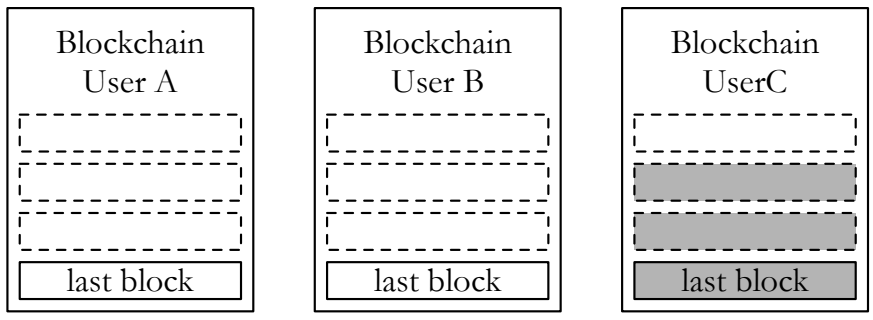

Fig. 2: Distributed blockchain.

of the message, that has fixed length, which is indicated in figure as hash. The security of a hash algorithm is that the function is not reversible (i.e. it cannot be traced back to the original message knowing only this data) and it should never be possible to intentionally create two different messages with the same digest. The digest to the SHA256 includes the blockID (for protecting from changes in the order of blocks), a nonce, the timestamp, the transaction(s) and a copy of the hash of the previous block. The nonce $n$ is specifically mined so that the resulting hash verifies specific conditions (e.g. it starts with a given number of zeros). In case one or more blocks get tampered, even in a single bit, the hash changes and the block is not considered valid anymore. With high probability, indeed, the condition on the hash is no more verified. A malicious user could mine the new nonce in order to obtain a valid block, however, its tampering is evident since the hash of such block is not equal to the corresponding hash in the blockchain owned by the majority of users. Besides, blocks are connected, namely chained, so that the hash of block i-1 is included as input to the SHA256 function to obtain the hash of the subsequent i-th block. In this way, any tamper on a block creates an invalid condition over all the following blocks in the chain, as shown in Fig. 2, where invalid blocks are indicated in gray, and the majority of users has a valid blockchain.

This chained setting has two effects: on one hand tampering a past block requires to mine nonces for all successive blocks (it is computationally unfeasible), on the other hand, the validity of the whole chain can be checked by verifying only the hash of the last block. The longer the blockchain is, the more difficult the change of the content as an effect of the computational burden for mining the nonces. A malicious user with large computational power able to mine all nonces would obtain a valid last block. However, even in such highly improbable case, the last hash would not match the one owned by the majority of the users, which are assumed as not colliding. In this largely simplified description, anyone knows about anyone elses transactions, exposing private data about energy generation and consumption. However, blockchains with confidential transactions have recently appeared [6] and provide a solution to such privacy concerns. Further details on the blockchain technology can be found in [7], while [8] provides a comprehensive presentation of the required cryptographic elements. 


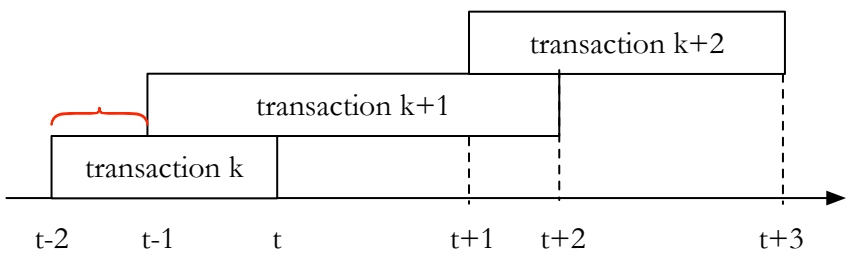

Fig. 3: Energy transactions cause the superposition of operating conditions.

\section{ENERGY BLOCKCHAIN FOR MICROGRIDS}

The blockchain technology in the energy field is becoming a realistic perspective, especially in energy districts. The issue, from a technical point of view, poses some challenges that are here analyzed. It is well-known that an energy transaction between a generator and a load may not correspond to the physical situation that appears applying power flow tracing methods. This is true for transmission systems [9] but also for small systems, where the presence of distributed generation with voltage support systems (PV nodes) implies a large circulation of reactive power. Therefore, after the transaction is started, a physical feasibility check is executed, to verify whether the considered transaction is or not viable in the current system. So, referring to Fig. 3, the transaction at block $\mathrm{k}$ implies generator $\mathrm{m}$ selling power to load $\mathrm{n}$.

After some time, a new transaction starts and the network hosts a new flow between generator $\mathrm{n}$ and load $\mathrm{f}$. This transaction causes, if the tracing algorithm indicates a superposition of the paths between generators and loads, for the time interval indicated in red in Fig. 3, a non-linear coupling of flows. In this way, a new distribution of power flows can either increase or reduce the efficiency if the distribution system in terms of losses. This idea is based on the following assumption: for each new transaction between a generator node and a load node, the generator involved is considered as a slack bus and the other generator nodes are set as P-V buses, the following steps can be taken:

1) identify the set points of the generators that are not taking part to the new energy transaction and set them to $\mathrm{P}-\mathrm{V}$ nodes with the value they had in the preceding block;

2) increase the load of the specified amount in $\mathrm{kW}$;

3) solve the load flow setting the generator involved in the new transaction as slack bus;

4) perform power flows tracing and identify the contribution of each generator to loads and active losses in each branch;

5) quantify the increase/reduction of active losses for the new energy transaction.

One of the main steps of the verification procedure is thus the power flow tracing. The active power flow tracing methods are well-established tools for analyzing the electric transmission networks because they allow to identify the contribution of every generation unit to the branch power flows on power lines. These methods can be applied after the load flow

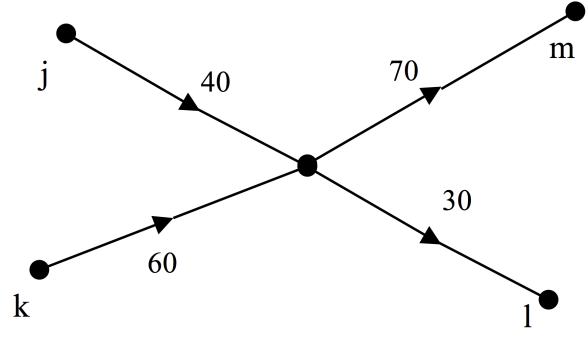

Fig. 4: Proportional sharing rule.

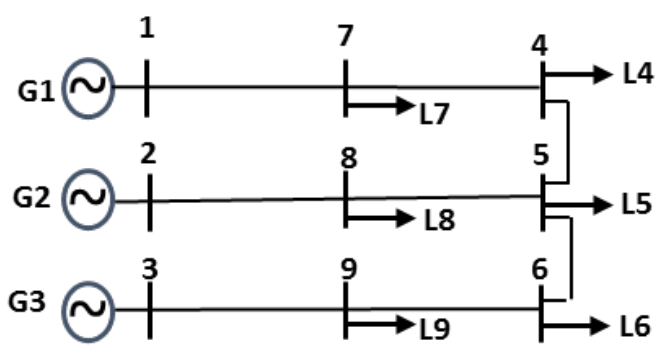

Fig. 5: Test system and topology of networks A and B.

solution and thus for each experiment a set of hypotheses about generation and loads should be done beforehand. The state of the art in power systems shows many papers that define algorithms for tracing the power flow; all are based on proportional sharing assumption [21]. According to this principle, given a node $i$ of the system, the contribution of every inflow, that involves an incoming line $j$, to every outflow megawatt, circulating on an outgoing line, is proportional to the ratio of power incoming from line $j$ and the total power flow through the $i$-th node [21]. In Fig. 4, a simple case of proportional sharing application on outgoing flows is presented.

In this way, the following calculations can be done:

$$
\begin{aligned}
P_{m}(j) & =\frac{70 \cdot 40}{60+40}=28 ; & P_{m}(k) & =\frac{70 \cdot 60}{60+40}=42 \\
P_{l}(j) & =\frac{30 \cdot 40}{60+40}=12 ; & P_{l}(k) & =\frac{30 \cdot 60}{60+40}=18
\end{aligned}
$$

$P_{m}(j), P_{l}(j), P_{m}(k), P_{l}(k)$ indicate the contribution of inflows (line $\mathrm{j}$ and $\mathrm{k}$ ) to the outflows (line $\mathrm{m}$ an $\mathrm{l}$ ). The sharing principle proposed above is widely adopted in most solution approaches of the power flows tracing problem [9][27]. However, all the referenced methods need the following input data as a result of the load flow calculation: power consumption at every load; power injection of every generation unit connected to the grid; power flow of every line and every transformer (both of them are seen as branches of a graph representation of the power grid).

\section{EXPERIMENTAL VALIDATION}

In this application, the small 9-bus MV system in Fig. 5 is considered. The purpose of the application is to compare two 


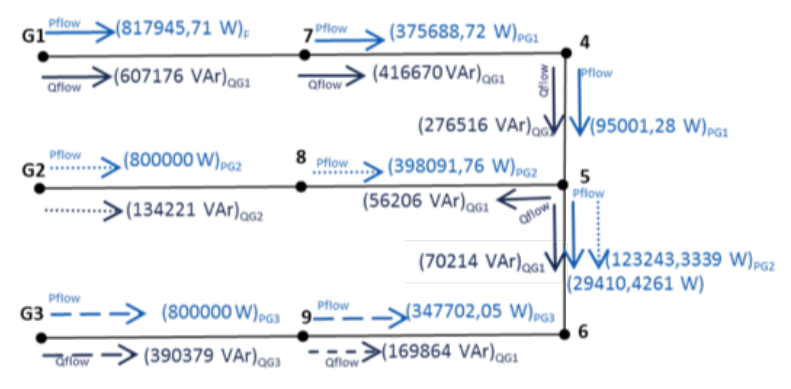

(a)

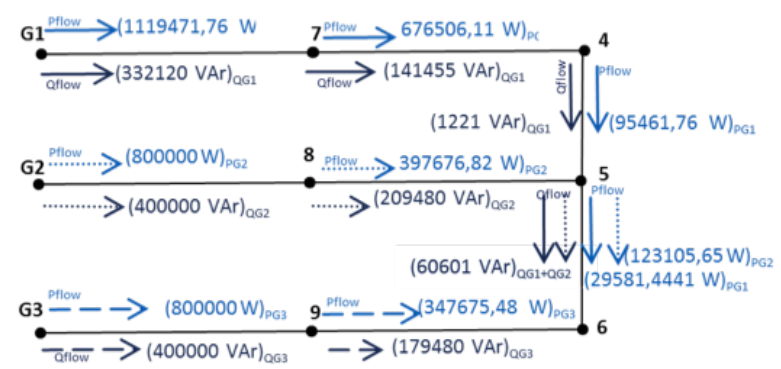

(c)

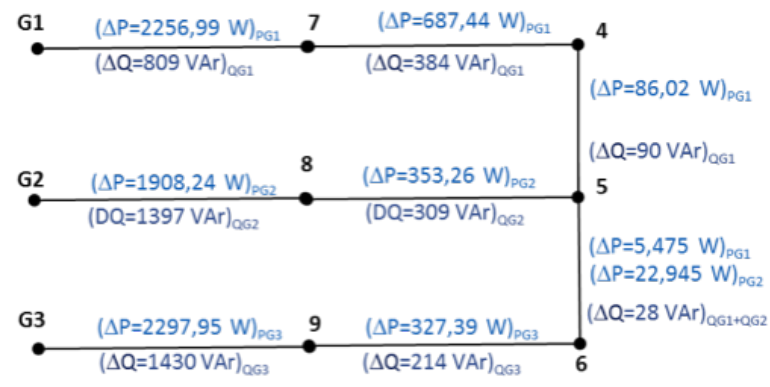

(b)

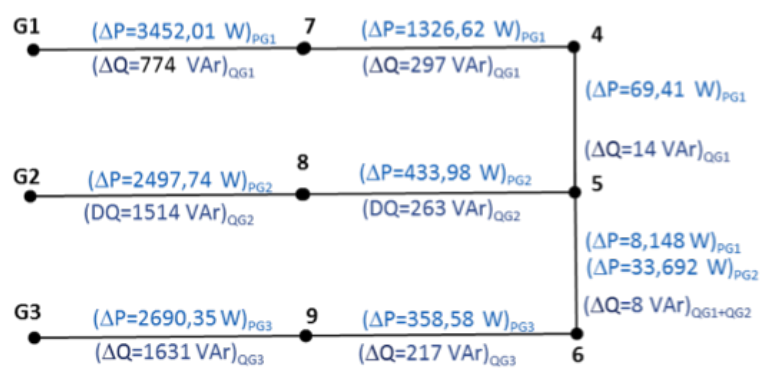

(d)

Fig. 6: Active and Reactive power computed on network A. Power flow tracing starting case (a), losses in branches for starting case (b), tracing case 1 (c), and power flow losses in branches in case 1.

TABLE I: Electrical features of network A: cable lines

\begin{tabular}{llll}
\hline branch & $\mathrm{R}[\Omega]$ & $\mathrm{X}[\Omega]$ & $\mathrm{B}[\Omega]^{-1}$ \\
\hline $1-7$ & 0.87 & 0.195 & 0.00009896 \\
$2-8$ & 1.16 & 0.2601 & 0.000132 \\
$3-9$ & 1.16 & 0.2601 & 0.000132 \\
$4-5$ & 0.406 & 0.09104 & 0.00004618 \\
$4-7$ & 0.87 & 0.195 & 0.00009896 \\
$5-6$ & 0.406 & 0.09104 & 0.00004618 \\
$5-8$ & 0.87 & 0.195 & 0.00009896 \\
$6-9$ & 0.87 & 0.195 & 0.00009896 \\
\hline
\end{tabular}

microgrids during an energy transaction based on blockchain technology. For network A, lines are all cables; for network $\mathrm{B}$, they are mixed aerial and cables. The comparison shows that:

- the involved reactive flows are huge and are largely influenced by the infrastructure and active power generation from PV buses

- the losses vary at every energy transaction

- it is possible to attribute losses to each energy transaction in real time by means of proportional sharing.

In tables I and II, the electrical features of the MV networks A and B are given. Three generators supply 6 residential loads. The generators can all provide voltage regulation by reactive support.

Therefore, we can summarize the whole results in table IV.

The active power of the PV buses is set to the value that was already running in the previous transaction and the reactive power staying within the limits of the generator. For each transaction we run first the algorithm for the power flow,
TABLE II: Electrical features of network B: aerial and cable lines

\begin{tabular}{llll}
\hline branch & $\mathrm{R}[\Omega]$ & $\mathrm{X}[\Omega]$ & $\mathrm{B}[\Omega]^{-1}$ \\
\hline $1-7$ & 0.87 & 0.195 & 0.00009896 \\
$2-8$ & 1.44 & 0.873 & 0.0000052 \\
$3-9$ & 1.44 & 0.873 & 0.0000052 \\
$4-5$ & 0.406 & 0.09104 & 0.00004618 \\
$4-7$ & 0.87 & 0.195 & 0.00009896 \\
$5-6$ & 0.406 & 0.09104 & 0.00004618 \\
$5-8$ & 1.08 & 0.654 & 0.0000039 \\
$6-9$ & 1.08 & 0.654 & 0.0000039 \\
\hline
\end{tabular}

TABLE III: Electrical features of the network in Fig. 4: nodes

\begin{tabular}{llllll}
\hline bus & type & $\begin{array}{l}\text { PG } \\
{[\mathrm{MW}]}\end{array}$ & $\begin{array}{l}\text { QGmin-QGmax } \\
{[\mathrm{MVAr}]}\end{array}$ & $\begin{array}{l}\text { PL } \\
{[\mathrm{MW}]}\end{array}$ & $\begin{array}{l}\text { QL } \\
{[\mathrm{MVAr}]}\end{array}$ \\
\hline 1 & slack & & & - & - \\
2 & $\mathrm{PV}$ & 0.8 & $-0.4 ; 0.4$ & - & - \\
3 & $\mathrm{PV}$ & 0.8 & $-0.4 ; 0.4$ & - & - \\
4 & $\mathrm{PQ}$ & - & - & 0.28 & 0.14 \\
5 & $\mathrm{PQ}$ & - & - & 0.34 & 0.15 \\
6 & $\mathrm{PQ}$ & - & - & 0.5 & 0.24 \\
7 & $\mathrm{PQ}$ & - & - & 0.44 & 0.19 \\
8 & $\mathrm{PQ}$ & - & - & 0.4 & 0.19 \\
9 & PQ & - & - & 0.45 & 0.22 \\
\hline
\end{tabular}

then for the flow tracing. Based on the proportional sharing principle, the partition coefficients are thus deduced. These coefficients give an indication about the contribution of each generator to the flow in each branch of the network and in the same way the amount of losses that can be attributed to each generator.

In table III, the loads and generation limits are also given. 


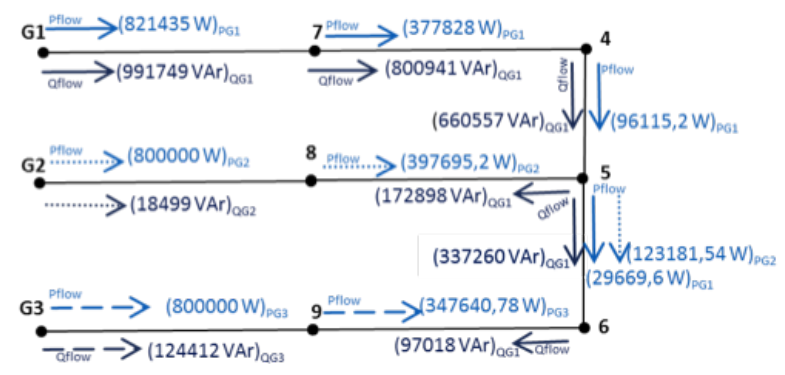

(a)

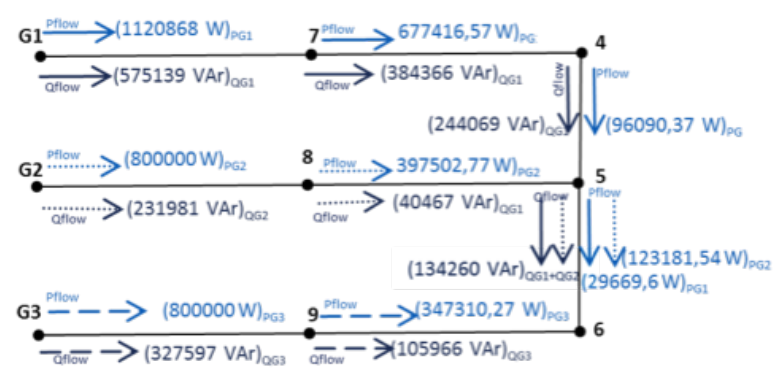

(c)

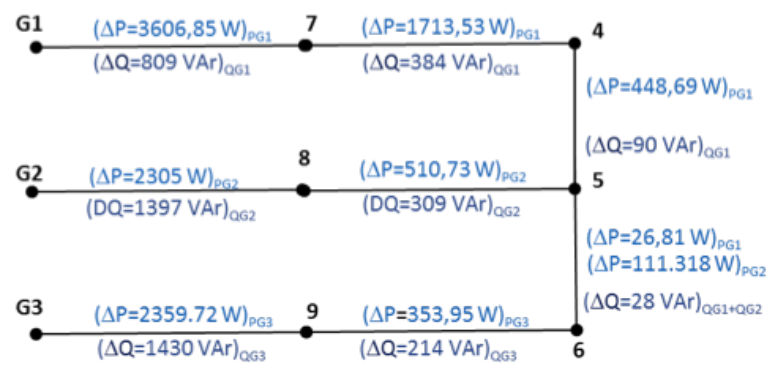

(b)

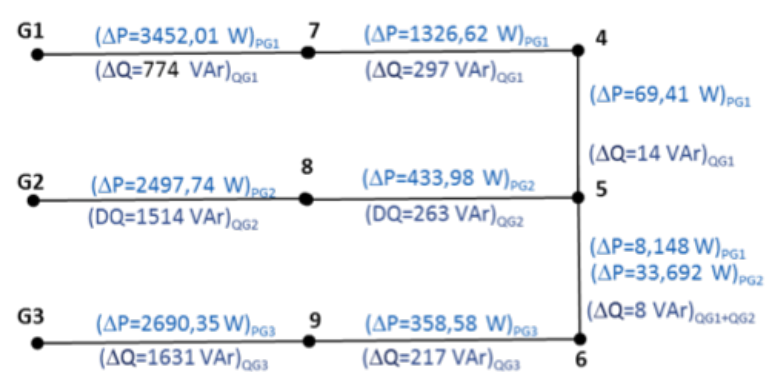

(d)

Fig. 7: Active and Reactive power computed on network B. Power flow tracing starting case (a), losses in branches for starting case (b), tracing case 1 (c), and power flow losses in branches in case 1 .

TABLE IV: Summary of results

\begin{tabular}{lllll}
\hline & Losses (PG1 supplying 4-7-5-6) & Losses (PG2 supplying 8-5-6) & Losses (PG3 supplying 9-6) & Overall Losses \\
\hline Case 0 network A & $\mathbf{3 0 3 5 , 9 2 5}$ & 2284,44457 & 2625,34 & 7945,71 \\
Case 1 network A & $\mathbf{4 0 2 4 , 4 7 4}$ & 2787,42642 & 2659,88 & 9471,78 \\
\hline & Losses (PG1 supplying 4-7-5-6) & Losses (PG2 supplying 8-5-6) & Losses (PG3 supplying 9-6) & Overall Losses \\
\hline Case 0 Network B & $\mathbf{5 7 7 7 , 2 5 9 2}$ & 2849,959 & 2809,612 & 11436,83 \\
Case 1 Network B & $\mathbf{4 8 5 6 , 1 8 7 6}$ & 2965,412 & 3048,93 & 10870,53 \\
\hline
\end{tabular}

The values given in table II are related to the starting state 0 and the first experiment is related to network A. For this state, the power flow problem is solved and the tracing algorithm is applied. The situation is depicted in Fig. 1, where the contribution of each generator to loads it is shown. Fig. 6b shows the contribution of each generator to active and reactive losses in each branch for the starting case. The arrows in the figure indicate the contribution of generators to Power Flow in the branches: arrows with continuous line indicate the contribution of G1; arrows with dotted line indicate the contribution of G2; arrows with dashed line indicate contribution of G3. Lets assume now (case 1) that the user at bus 4 wants to buy 300 $\mathrm{kWh}$ from bus 1 from 11:00 a.m. to 12:00 a.m. as depicted in Fig. $6 \mathrm{c}$ and d, for network A, and in Fig. 7 for network B.

As it appears from the proposed example, by means of the application of a tracing algorithm based on proportional sharing, a value of losses can be precisely referred to a given transaction. A second transaction would cause a further variation of losses in all active transactions that should be distributed among the generators that are involved in the transactions in the considered timeframe. The same transaction taking place in network B shows that an increase of active power implies a different behavior. As it appears from the proposed example, the game of reactive flows generates the following situation: a new active power delivery reliefs the system from losses and thus this situation should be accounted for in the economic evaluation of the relevant transaction. The situation is depicted in Fig. 7, where the contribution of each generator to loads and losses is shown, considering both the starting case and case 1 .

In the table, in bold is indicated the value of power losses associated to the flow between the generator (in the column) and the supplied loads that is changing (at bus 4). As it can be observed, the transaction that is established between the user at node 4 and the generator at node 1 , determines in general in the branches included in the path from the generator to the load an increase of power flows, compared to the previous situation. This changes the profile of the node voltages, and results in a redistribution of reactive power flows in the network branches following their electrical characteristics. In the case of network A, the effect is an overall increase in active power losses, which is distributed between the three generators supplying the network. As it can be seen from Fig. $7 \mathrm{c}$ and d, in the case of non-uniform network (B) the transaction between the 
load 4 and the generator G1 determines a reduction of active power losses in the branches of the path from the generator to the load. On the contrary, it the new transaction in this case generates an increase of losses in the branches powered from generators that are not directly involved in the transaction. Altogether, the total losses in the system are reduced. Different scenarios are expected for new overlapping transactions. For this reason the authors believe that an on-line attribution of power losses is required in such situations.

\section{CONCLUSIONS AND FUTURE WORK}

When a generator feeds a generic load in an islanded microgrid, a subsequent energy transaction superimposes the effects non-linearly, since additional losses couples non-linearly to the existing flows. In this paper, it is argued that the additional term related to the power losses could be shared according to the real physical situation at hand, based on the evaluation of losses and to their imputation to each generator.

Further developments of this work regard the possibility to deploy the tracing algorithm in a decentralized fashion and to finely attribute losses costs to users. The algorithm implemented in [29] performs a distributed power flow tracing, by which it is possible to identify the power supplying a given load and the associated losses for a given load flow configuration. Therefore, the distributed ledger could also be associated to a distributed computation that could verify the technical feasibility of the transaction between two nodes of the network.

\section{REFERENCES}

[1] Gartner, Gartner's 2016 Hype Cycle for Emerging Technologies Identifies Three Key Trends That Organizations Must Track to Gain Competitive Advantage, http://www.gartner.com/newsroom/id/3412017

[2] P. Frystad, J. Holm, Blockchain: Powering the Internet of Value, EVRY Labs, 2016.

[3] VANDEBRON ENERGIE B.V. https://vandebron.nl/

[4] D. Tapscott, A. Tapscott, Blockchain Revolution: How the Technology Behind Bitcoin Is Changing Money, Business, and the World, Penguin Publishing Group, 2016.

[5] H. Gilbert, H. Handschuh, Security Analysis of SHA-256 and Sisters, Lecture Notes in Computer Science, Vol. 3006, Selected Areas in Cryptography, pp. 175193, 2003.

[6] Pilkington, Marc, Blockchain Technology: Principles and Applications (September 18, 2015). Research Handbook on Digital Transformations, edited by F. Xavier Olleros and Majlinda Zhegu. Edward Elgar, 2016.

[7] Antonopoulos, Andreas M. Mastering Bitcoin: unlocking digital cryptocurrencies. " O'Reilly Media, Inc.", 2014.

[8] Menezes, Alfred J., Paul C. Van Oorschot, and Scott A. Vanstone. Handbook of applied cryptography. CRC press, 1996.

[9] L. Dusonchet, S. Favuzza, M. G. Ippolito, F. Massaro, G. Patern, Numerical implementation of active power flow tracing methods: practical implications on transmission networks and DR programs support, 2015 IEEE 15th Intl. Conf. on Environment and Electrical Engineering, pp. 531 - 536, 10-13 June 2015, Rome (Italy).

[10] J. Bialek, Trancing the flow of electricity, IEE Proc. Generation, Transmission and Distribution, Vol. 143, n.4, pp. 313- 320, 1996.

[11] D. Kirschen, R. Allan and G. Strnac, Contributions of individual generators to loads and flows, IEEE Transactions on Power Systems, vol. 12, issue 1, pp. 52-60, February 1997. doi: 10.1109/59.574923.

[12] F.F. Wu, Y. Ni and P. Wei, Power transfer allocation for open access using graph theory - Fundamentals and applications in systems without loop flow, IEEE Trans on Power Systems, vol. 15, issue 3, pp. 923- 929, August 2000. doi: 10.1109/59.871714.
[13] F.F. Wu, Y. Ni and P. Wei, Load flow tracing in power system with circulation power, Intl Journal of Electrical Power \& Energy Systems, vol. 24, issue 10, pp. 807813, December 2002. doi:10.1016/S01420615(02)00008-X.

[14] F.F. Wu, P. Wei, B. Yuan and Y. Ni, Power flow tracing for transmission open access, Proc of Intl Conf on Electric Utility Deregulation and Restructuring and Power Technologies pp. 476-481, April 2000. doi:10.1109/DRPT.2000.855711

[15] K. Xie, J. Zhou and W. Li, Analytical model and algorithm for tracing active power flow based on extended incidence matrix, Electric Power System Research, vol. 79, issue 2, pp. 399-405, February 2009. doi: 10.1016/j.epsr.2008.08.001

[16] M.W. Mustafa and H.Shareef, A Comparison of Electric Power Tracing Methods Used in Deregulated Power Systems, IEEE International Power and Energy Conference, pp. 156-160, November 2006 doi:10.1109/PECON.2006.346638

[17] A. Augugliaro, L. Dusonchet, S. Favuzza, M.G. Ippolito and E. Riva Sanseverino, A model for reactive power tracing by addition of fictitious nodal injections, Electric Power Systems Research, vol. 83, issue 1, pp. 196202, February 2012. doi:10.1016/j.epsr.2011.11.006.

[18] A. Augugliaro, L. Dusonchet, S. Favuzza, M.G. Ippolito and E. Riva Sanseverino, Influence of losses partition criteria on power flow tracing, IEEE Intl Energy Conference and Exhibition, pp. 310317, September 2012. doi:10.1109/EnergyCon.2012.6347773.

[19] J.C. Peng and H. Jiang, Contributions of individual generators to complex power losses and flows. Fundamental theory, IEE ProceedingsGeneration, Transmission and Distribution, vol. 149, issue 2, pp. 182185 , March 2002. doi:10.1049/ip-gtd:20020094.

[20] Z. Jing and F. Wen, Discussion on the Proving of Proportional Sharing Principle in Electricity Tracing Method, Transmission and Distribution Conference and Exhibition: Asia and Pacific, pp. 1-5, 2005. doi:10.1109/TDC.2005.1547035.

[21] J. Bialek, Topological generation and load distribution factors for supplement charge allocation in transmission open access, IEEE Trans on Power Systems, vol. 12, issue 3, pp. 1185-1193, August 1997. doi:10.1109/59.630460.

[22] D. Kirschen and G. Strbac, Tracing active and reactive power between generators and loads using real and imaginary currents, IEEE Trans on Power Systems, vol. 14, issue 4, pp. 1312-1319, November 1999. doi:10.1109/59.801890.

[23] Z. Ming, S. Liyng, L. Gengyin and Y. Ni, A novel power flow tracing approach considering power losses, IEEE International Conference on Electric Utility Deregulation, Restructuring and Power Technologies, vol. 1, pp. 355-359, April 2004. doi:10.1109/DRPT.2004.1338521.

[24] Z. Qu, J. Qin, X. Wang, H. Bao and C. Zhou, A Power Flow Tracing Method Based on the Circuit Analysis of Power Supply Path, Power and Energy Engineering Conference, Asia-Pacific, pp.1-6, March 2011. doi: 10.1109/APPEEC.2011.5748832.

[25] F. Gubina, D. Grgic and I. Banic, A method for determining the generators' share in a consumer load, IEEE Transactions on Power Systems, vol. 15, issue 4, pp. 1376-1381, November 2000. doi:10.1109/59.898115.

[26] C. Li, D. Wu and Y. Guizhong, The research of power flow tracing analytical algorithm, China International Conference on Electricity Distribution, pp. 1-4, September 2012. doi:10.1109/CICED.2012.6508574.

[27] H. Sun, D.C. Yu and Q. Zheng, AC power flow tracing in transmission networks, IEEE Power Engineering Society Winter Meeting, vol. 3, pp. 1715-1720, January 2000. doi: 10.1109/PESW.2000.847609.

[28] S.P. Rosado, K. Abdel-Rahman and N. Hadjsaid, Tracing the path of electric power flow-a study for deregulated power systems, IEEE Power Engineering Society Winter Meeting, vol. 3, pp. 1479-1484, January 2001. doi:10.1109/PESW.2001.917327.

[29] Bracco Stefano, Buono Luca, Delfino Federico, Di Silvestre Maria Luisa, Riva Sanseverino Eleonora, Distributed Optimal Power Flow for Islanded Microgrids: an Application to the Smart Polygeneration Microgrid of The Genoa University, In Proceedings of 2nd International Conference on Smart Cities (ISC2), Trento, 12-15 settembre 2016. 